\title{
Editorial
}

\section{Treatment with intravenous bisphosphonates in children and adolescents with Osteogenesis Imperfecta: are we towards a consensus of a protocol?}

\author{
Konstantinos D. Stathopoulos M.D., Ph.D. \\ Postgraduate Course on Metabolic Bone Disorders, National and Kapodistrian University of Athens, School of Medicine, Greece
}

In the current issue of JRPMS, Dr Sarantis and colleagues report the interesting case of a Greek patient, a 7 years-old boy, with Osteogenesis Imperfecta due to a novel COL1A1 mutation'. The authors also provide insight into their protocol of pharmacological treatment with iv zolendronic acid, a topic of increasing interest in the field, as there is currently no consensus regarding choice of pharmacological agent, dosing or even duration of treatment.

Osteogenesis Imperfecta (OI) literally means "imperfect bone birth" in Latin, as derived from the original Greek

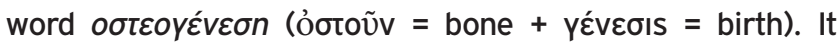
was termed by Vrolik in 1849 and represents a genetic disorder with multiple genotypes and phenotypes. Ol is caused by mutations either of the COL1A1 or COL $1 \mathrm{~A} 2$ genes (in reportedly $85 \%$ of the cases) encoding the pro-a 1 or a 2 chains of type-I collagen or of other genes (i.e WNT1, LRP5, BMP 1, CRTAP, P3H1/LEPRE 1) involved in osteoblast differentiation or post-translational modification/transport of type I collagen ${ }^{2-4}$. Type-I collagen is the major protein of bone, constituting by large its organic part, but exists also in significant quantities in tendons, ligaments, skin, sclerae and dentin. Patients with OI have lower quantity and/or quality of type-I collagen, thus presenting with multiple clinical phenotypes that usually include low-energy fractures, bone deformities, joint hypermobility, bone pain, short stature, and in some cases blue sclerae, dentinogenesis imperfecta and premature hearing loss. According to the original classification of Sillence ${ }^{5}$, Ol can be distinguished in four predominant types (I-IV). Type I is the milder form with usually no deformities, type II is the lethal form resulting in perinatal death, type III is the most severe in surviving neonates with multiple fractures and deformities, and type IV is intermediate in severity between types I and III with moderate deformities and short stature.

Pharmacological treatment of $\mathrm{OI}$ in various clinical settings reportedly depends upon the age of the patient at the time of diagnosis as well as the severity of the disease, and there is currently no consensus regarding a treatment regimen of choice ${ }^{4}$. In the past, unsuccessful attempts to control the disease have been made with vitamins, sodium fluoride, calcitonin or even growth hormone. Twenty years ago, Glorieux and colleagues reported the use of intravenous pamidronate (a second-generation nitrogen-containing bisphosphonate) in children with severe osteogenesis imperfecta ${ }^{6}$. Ever since, there have been a few reports regarding the short-term effects of pamidronate treatment in various dosing regimens for sometimes up to 4 years in small numbers of patients with $\mathrm{Ol}^{6-10}$. These studies, comprising groups of children with OI types I, III and IV all reported significant increases in lumbar spine areal bone mineral density (BMD). One study reported beneficial effects on lumbar spine BMD during treatment for 2-9 years ${ }^{9}$. A very interesting study with iliac bone histomorphometry ${ }^{10}$ in 45 patients with Ol suggested that after 2 years of treatment, iv pamidronate in different doses and dose regimens according to the age, induced significant increases in cortical width $(88 \%, p<0.001)$ and average cancellous bone volume $(46 \%, p<0.001)$ that was entirely due to an increase in trabecular number, while trabecular thickness remained unchanged. Bone formation rate (BFR/BS), osteoid thickness, and other markers of trabecular bone remodelling all significantly decreased $(p<0.001)$, and the authors concluded that pamidronate has a 2 -fold effect on the growing skeleton, by inhibiting bone remodelling-induced trabecular bone resorption while preserving bone formation of cortical bone due to bone modelling ${ }^{10}$. One study reported that iv pamidronate given for 4 years led to a statistically

The author has no conflict of interest.

Corresponding author: Konstantinos D. Stathopoulos M.D., Ph.D., Postgraduate Course on Metabolic Bone Disorders, National and Kapodistrian University of Athens, School of Medicine, Greece

E-mail: kossta51@hotmail.com

Accepted 28 August 2018 
significant increase in height in patients with Ol type IV ${ }^{11}$. Very few studies were performed with nerindronate, another iv bisphosphonate, in children and adolescents with $\mathrm{Ol}^{12-13}$. Idolazzi et al reported significant increases of areal BMD at the lumbar spine and total hip versus baseline but no statistically significant effect on fracture risk $(p=0.185)$, although they report a significant reduction in the mean number of fractures occurring during treatment compared to pre-treatment values ${ }^{12}$. Gatti et al also reported significant increases of lumbar and hip BMD but no significant effect on fracture incidence ${ }^{13}$.

After 2003 , and especially during the last 10 years, zolendronic acid (ZOL), a third-generation nitrogencontaining iv bisphosphonate has been increasingly used in the treatment of OI. ZOL is thought to possess multiple advantages over pamidronate as it has higher potency, a very short infusion time of 15-30 minutes (as opposed to 2-4 hours for pamidronate, which was administered usually in 2-3 consecutive days), is reported to reduce incidence of phlebitis due to lower infusion time, and has a longer duration of action, meaning that infusions can be performed once or twice a year while pamidronate may be administered 2-6 times a year depending on the patient's age ${ }^{14}$. Concerning dosage, initial and subsequent doses range between 0.025$0.05 \mathrm{mg} / \mathrm{kg}$ selected for each patient on individual basis. Panigrahi et al studied 5 Ol type III children aged 0-7y that were treated with $\mathrm{ZOL}$ for an average of 20 months and reported significant increases in areal BMD and decreased rate of fractures $(P=0.002)^{15}$. Vuorimies at al studied 17 children with OI type I that were treated for 1.0-3.2 years with $0.05 \mathrm{mg} / \mathrm{kg}$ ZOL iv every 6 months and reported increases in lumbar $\mathrm{BMD}^{16}$. Barros et al performed a randomised control trial of 1 year treatment with either ZOL $(0.025-0.05 \mathrm{mg} /$ $\mathrm{kg} /$ day for 2 days) or pamidronate ( $1 \mathrm{mg} / \mathrm{kg} /$ day for 2 days) every 3-4 months according to their ages in children with Ol and reported marginally statistically significant increases of spine BMD in the ZOL group ${ }^{17}$. Otaify et al reported also significant increases of lumbar and BMD in children with OI treated with ZOL $(0.1 \mathrm{mg} / \mathrm{kg}$ every 6 months for 2 years) with a significant decrease in fracture rate, bone pain and improvement in ambulation ${ }^{18}$. Finally, Palomo et al reported long-term outcomes in patients with $\mathrm{Ol}$ who received iv treatment with bisphosphonates (pamidronate or $\mathrm{ZOL}$ ) in their early childhood and were followed for up to 18 years (median 14.8y) ${ }^{19}$. According to their results, lumbar BMD increased, height significantly increased only in patients with type IV, only $6 \%$ of vertebrae appeared compressed at the last evaluation (vs $35 \%$ at baseline, $p<0.001$ ) but fracture incidence of long bones was still high.

It is evident that randomised clinical trials and reporting on long term results, fracture incidence, height development, scoliosis, and functional outcomes are largely missing in children and adolescents with OI treated with iv bisphosphonates. There are still questions to be answered, concerning optimum time of onset, dosing and duration of treatment, dosing interval or drug holiday or discontinuation. However, all relevant research suggests that treatment with bisphosphonates is beneficial for patients with OI. If a consensus protocol were to be introduced by a panel of specialists with experience in the treatment of children with Ol, more data from clinical settings worldwide would be added so that we can address these questions more adequately as a scientific community in the interest of patients.

\section{References}

1. Sarantis M, Kollia P, Samara S, Athanasopoulou H, Gyftodimou Y, Lianou D, Mpourazani E, Doulgeraki A. A new COL 1 A 1 mutation in a Greek patient with osteogenesis imperfecta: Response to a lowdose protocol of zoledronic acid and two-year follow-up. JRPMS 2018;2(3):75-80.

2. Rauch F, Travers R, Plotkin H, Glorieux F.H. The effects of intravenous pamidronate on the bone tissue of children and adolescents with osteogenesis imperfecta. J Clin Invest 2002; 1 10: 1293-1299.

3. Morello R. Osteogenesis Imperfecta and therapeutics. Matrix Biol 2018; pii:S0945-053X(18)30050-7.

4. Dwan K, Phillipi CA, Steiner RD, Basel D. Bisphosphonate therapy for osteogenesis imperfecta. Cochrane Database of Systematic Reviews 2014;23(7):CDOO5088.

5. Sillence DO, Senn A, Danks DM. Genetic heterogeneity in osteogenesis imperfecta. J Med Genet 1979;16:101-116.

6. Glorieux FH, Bishop NJ, Plotkin H, Chabot G, Lanoue G, Travers R. Cyclic administration of pamidronate in children with severe osteogenesis imperfecta. N Engl J Med 1998;339:947-52.

7. Plotkin H, Rauch F, Bishop N.J. et al. Pamidronate treatment of severe osteogenesis imperfecta in children under 3 years of age. $J$ Clin Endocrinol Metab 2000;85:1846-50.

8. Arikoski P, Silverwood B, Tillmann V, Bishop N,J. Intravenous pamidronate treatment in children with moderate to severe osteogenesis imperfecta: assessment of indices of dual-energy $X$-ray absorptiometry and bone metabolic markers during the first year of therapy. Bone 2004;34(3):539-46.

9. Astrom E, Soderhall S. Beneficial effect of long term intravenous bisphosphonate treatment of osteogenesis imperfecta. Arch Dis Child 2002;86(5):356-64.

10. Rauch F, Travers R, Plotkin H, Glorieux FH. The effects of intravenous pamidronate on the bone tissue of children and adolescents with osteogenesis imperfecta. J Clin Invest 2002; 1 10:1293-9.

11. Zeitlin L, Rauch F, Plotkin H, Glorieux FH. Height and weight development during four years of therapy with cyclical intravenous pamidronate in children and adolescents with osteogenesis imperfecta types I, III, and IV. Pediatrics 2003; 1 11:1030-6.

12. Idolazzi L, Fassio A, Viapiana O, Rossini M, Adami G, Bertoldo F, Antoniazzi F, Gatti D. Treatment with neridronate in children and adolescents with osteogenesis imperfecta: Data from open-label, not controlled, three-year Italian study Bone 2017;103:144-149.

13. Gatti D, Antoniazzi F, Prizzi R, Braga V, Rossini M, Tato L, et al. Intravenous neridronate in children with osteogenesis imperfecta: $A$ randomized controlled study. Journal of Bone and Mineral Research 2005;20(5):758-63.

14. George S, Weber DR, Kaplan P, Hummel K, Monk HM, Levine MA. Short-Term Safety of Zoledronic Acid in Young Patients With Bone Disorders: An Extensive Institutional Experience. J Clin Endocrinol Metab 2015; 100:4163-4171

15. Panigrahi I, Das RR, Sharda S, Marwaha RK, Khandelwal N. Response 
to zolendronic acid in children with type III osteogenesis imperfecta. J Bone Miner Metab 2010;28(4):451-455.

16. Vuorimies I, Toiviainen-Salo S, Hero M, Makitie O. Zolendronic acid treatment in children with osteogenesis imperfecta. Horm Res Pediatr 2011;75(5):346-53.

17. Barros ER, Saraiva GL, de Oliveira TP, Lazaretti-Castro M. Safety and efficacy of a 1-year treatment with zoledronic acid compared with pamidronate in children with osteogenesis imperfecta. J Pediatr Endocrinol Metab 2012;25(5-6):485-91.
18. Otaify GA, Aglan MS, Ibrahim MM, Elnashar M, El Banna RA, Temtamy SA. Zoledronic acid in children with osteogenesis imperfecta and Bruck syndrome: a 2-year prospective observational study. Osteoporos Int 2016;27(1):81-92.

19. Palomo T, Fassier F, Ouellet J, Sato A, Montpetit K, Glorieux $\mathrm{FH}$, Rauch F. Intravenous Bisphosphonate Therapy of Young Children With Osteogenesis Imperfecta: Skeletal Findings During Follow Up Throughout the Growing Years. J Bone Miner Res 2015;30(12):2150-7. 